\title{
Search and Preliminary Analysis of New Galactic PNe
}

\author{
C. Zanin $^{1,2}$, E. Cappellaro ${ }^{3}$, F. Sabbadin ${ }^{3}$ and M. Turatto ${ }^{3}$ \\ ${ }^{1}$ Dipartimento di Astronomia, Padova, Italy; ${ }^{2}$ Institut für Astronomie,Innsbruck, Austria ; \\ ${ }^{3}$ Osservatorio Astronomico di Padova, Italy
}

In general, the methods for searching new PNe exploit one of their characteristics and typically introduce a bias in the sample extracted. It is therefore important to perform PN searches based on different selection criteria. Some years ago we started a new search for PN candidates on the plates of the Palomar Near Infrared Photographic Survey of the Galactic Plane. The selection criterion is based on the consideration that PNe have $I(R)>$ $I(N I R)$ up to $A_{V} \sim 10$, whereas normal field stars have $I(R)<I(N I R)$. First the plates are examined directly using a binocular, with a medium magnification, searching for faint diffuse nebulosity. Hereafter the comparison between R and NIR images is used both to detect new compact PN candidate and to discard, among diffuse objects, reflection nebulae. Spectra and imaging of the new objects were obtained in dedicated program both at the ESO and Asiago Observatories. From a preliminary analysis of the data for the new PNe it appears that the sample contains objects with very different characteristics. They have different morphologies and spectral features, but in general only few of the new PNe have an IRAS counterpart and these show a small flux in the IR. In particular, in the IRAS color-color plot they do not seem confined to the area of the bona-fide PNe; this still preliminary evidence may indicate that our selection criteria is pointing to $\mathrm{PNe}$ which remain undiscovered using other methods.

The list of the new PNe discovered so far is given in the table where we report the name of the objects, their PN Glll.1 \pm bb.b identification (Acker et al., 1992, Strasbourg-ESO Catalogue of Galactic PNe, ESO Munich, Germany) and their equatorial coordinates.

\begin{tabular}{|c|c|c|c|c|c|c|c|c|c|c|c|c|c|c|c|}
\hline \multirow{2}{*}{$\begin{array}{l}\text { Name } \\
\text { Sab38 }\end{array}$} & \multirow{2}{*}{$\begin{array}{l}\text { PNG } \\
000.7-06.1\end{array}$} & \multirow{2}{*}{$\frac{\alpha}{18}$} & \multicolumn{2}{|c|}{$(\mathrm{J} 2000.0)$} & \multicolumn{3}{|l|}{$\delta$} & \multirow{2}{*}{$\begin{array}{l}\text { Name } \\
\text { NeVe } 4^{b}\end{array}$} & PNG & $\alpha$ & \multicolumn{2}{|c|}{$(\mathrm{J} 2000.0)$} & \multicolumn{3}{|l|}{$\bar{\delta}$} \\
\hline & & & 12 & 14.1 & -31 & 19 & $59^{a}$ & & $169.8+01.9$ & 05 & 27 & 11 & +38 & 31 & 30 \\
\hline $\begin{array}{l}\text { Sab37 } \\
\text { Sab24 }\end{array}$ & $\begin{array}{l}000.6-05.3 \\
001.4+06.3\end{array}$ & $\begin{array}{l}18 \\
17\end{array}$ & $\begin{array}{l}08 \\
24\end{array}$ & $\begin{array}{l}35.1 \\
53.4\end{array}$ & $\begin{array}{l}-31 \\
-24\end{array}$ & $\begin{array}{l}06 \\
19\end{array}$ & $\begin{array}{l}53^{a} \\
29^{a}\end{array}$ & $\begin{array}{l}\text { Sab13ir } \\
\text { NeVe14 }\end{array}$ & $\begin{array}{l}212.6-00.0 \\
250.4-01.3\end{array}$ & $\begin{array}{l}06 \\
08\end{array}$ & $\begin{array}{l}50 \\
03\end{array}$ & $\begin{array}{l}42.0 \\
12.7\end{array}$ & $\begin{array}{l}+00 \\
-33\end{array}$ & $\begin{array}{l}14 \\
30\end{array}$ & $\begin{array}{l}26 \\
30\end{array}$ \\
\hline Sab44 & $001.6-00.5$ & 17 & 51 & 52.9 & -27 & 47 & 42 & NeVe15 & $251.1+00.7$ & 08 & 13 & 20.6 & -33 & 01 & 07 \\
\hline Sab25 & $002.0+06.6$ & 17 & 25 & 41.6 & -23 & 38 & $29^{a}$ & NeVe22 & $275.9-01.0$ & 09 & 34 & 04.6 & -53 & 12 & 24 \\
\hline Sab59 & $002.2-01.2$ & 17 & 55 & 45.7 & -27 & 39 & $35^{a}$ & NeVe24 & $280.5+01.7$ & 10 & 10 & 34.4 & -53 & 56 & 31 \\
\hline Sab27 ${ }^{b}$ & $002.4+01.1$ & 17 & 46 & 52 & -26 & 13 & 04 & $\mathrm{NeVe} 26^{2 r}$ & $288.0-01.6$ & 10 & 44 & 38.0 & -60 & 47 & 47 \\
\hline $\operatorname{Sab} 30^{b}$ & $004.1+07.8$ & 17 & 26 & 11 & -21 & 17 & 34 & NeVe33 & $298.4+02.4$ & 12 & 15 & 30.9 & -60 & 08 & 41 \\
\hline $\begin{array}{l}\text { Sab15 }{ }^{b} \\
\text { Sab31 }\end{array}$ & $\begin{array}{l}006.8+05.0 \\
008.8+03.8\end{array}$ & $\begin{array}{l}17 \\
17\end{array}$ & $\begin{array}{l}42 \\
51\end{array}$ & $\begin{array}{l}24 \\
08.3\end{array}$ & $\begin{array}{l}-20 \\
-19\end{array}$ & $\begin{array}{l}26 \\
25\end{array}$ & $\begin{array}{l}23 \\
45^{a}\end{array}$ & $\begin{array}{l}\text { Sab81 } \\
\text { Sab82 }\end{array}$ & $\begin{array}{l}340.9+03.7 \\
341.7+02.6\end{array}$ & $\begin{array}{l}16 \\
16\end{array}$ & $\begin{array}{l}34 \\
42\end{array}$ & $\begin{array}{l}50 \\
11\end{array}$ & $\begin{array}{l}-42 \\
-42\end{array}$ & $\begin{array}{l}03 \\
14\end{array}$ & $\begin{array}{l}45 \\
41\end{array}$ \\
\hline Sab12 & $009.8-01.1$ & 18 & 11 & 39 & -21 & 01 & 16 & Sab70 & $345.4-02.3$ & 17 & 15 & 46.9 & -42 & 24 & $05^{a}$ \\
\hline Sab16. & $009.9+04.5$ & 17 & 50 & 46.9 & -18 & 03 & $30^{a}$ & Sab74 & $347.4+01.8$ & 17 & 04 & 16.7 & -38 & 19 & $55^{a}$ \\
\hline Sab88 ir & $010.0-01.4$ & 18 & 13 & 29.3 & -20 & 57 & 08 & Sab83 & $348.9+04.6$ & 16 & 57 & 59 & -35 & 24 & 35 \\
\hline Sab21 ir & $010.1+07.4$ & 17 & 41 & 04 & -16 & 24 & $29^{a}$ & Sab61/2b & $351.1-03.9$ & 17 & 39 & 17 & -38 & 29 & 51 \\
\hline Sab17ir & $010.2+02.7$ & 17 & 58 & 14 & -18 & 42 & 14 & $S a b 42^{b}$, ir & $351.4-03.3$ & 17 & 37 & 28.6 & -37 & 59 & $19^{a}$ \\
\hline$S a b 32^{b}$ & $010.2+00.3$ & 18 & 06 & 53 & -19 & 55 & 36 & Sab66 ${ }^{b}$ & $353.4-04.5$ & 17 & 48 & 00.7 & -36 & 50 & $06^{a}$ \\
\hline Sab34ir & $011.0-02.9$ & 18 & 20 & 52 & -20 & 47 & 35 & Sab67 $b$ & $354.4+05.4$ & 17 & 10 & 43.1 & -30 & 32 & $26^{a}$ \\
\hline Sab8 $6^{b}$ & $011.2-02.7$ & 18 & 20 & 31 & -20 & 30 & 37 & Sab50 & $354.4+03.2$ & 17 & 19 & 01.8 & -31 & 47 & $36^{a}$ \\
\hline Sab39ir & $011.7+00.2$ & 18 & 10 & 19 & -18 & 39 & 21 & Sab63 ${ }^{i r}$ & $354.5-01.7$ & 17 & 38 & 54.3 & -34 & 27 & $36^{a}$ \\
\hline Sab11 ir & $011.7-04.9$ & 18 & 30 & 08.5 & -21 & 04 & 55 & Sab41 & $354.5-03.9$ & 17 & 48 & 16.1 & -35 & 38 & $35^{a}$ \\
\hline $\begin{array}{l}\text { Sab33 } \\
\text { Sab10 }\end{array}$ & $\begin{array}{l}012.1-02.1 \\
012.5+04.3\end{array}$ & $\begin{array}{l}18 \\
17\end{array}$ & $\begin{array}{l}20 \\
57\end{array}$ & $\begin{array}{l}17.3 \\
12.9\end{array}$ & $\begin{array}{l}-19 \\
-15\end{array}$ & $\begin{array}{l}26 \\
56\end{array}$ & $\begin{array}{l}38 \\
18\end{array}$ & $\begin{array}{l}\text { NeVe18 } \\
\text { Sab64 }\end{array}$ & $\begin{array}{l}355.1-15.9 \\
355.6-02.3\end{array}$ & $\begin{array}{l}18 \\
17\end{array}$ & $\begin{array}{l}43 \\
44\end{array}$ & $\begin{array}{l}57 \\
27.2\end{array}$ & $\begin{array}{l}-40 \\
-33\end{array}$ & $\begin{array}{l}29 \\
55\end{array}$ & $\begin{array}{l}56 \\
01^{a}\end{array}$ \\
\hline $\mathrm{Sab7} 7^{i r}$ & $019.2-04.4$ & 18 & 42 & 25.5 & -14 & 15 & 02 & Sab40 & $356.4-02.5$ & 17 & 47 & 12.2 & -33 & 16 & $25^{a}$ \\
\hline Sab8. & $024.8-01.4$ & 18 & 41 & 48 & -07 & 51 & 04 & Sab49ir & $356.5+02.2$ & 17 & 28 & 06.7 & -30 & 38 & $18^{a}$ \\
\hline Sab6 ir & $026.2-03.4$ & 18 & 51 & 32.5 & -07 & 32 & 22 & Sab43 & $357.5-02.4$ & 17 & 49 & 35.9 & -32 & 16 & 52 \\
\hline NeVe12 ${ }^{b}$ & $073.0+03.6$ & 20 & 00 & 19 & +36 & 59 & 19 & Sab4 $7^{b, i r}$ & $357.8+01.6$ & 17 & 33 & 59 & -29 & 55 & 00 \\
\hline NeVe13 ${ }^{b}$ & $079.7+00.4$ & 20 & 33 & 13 & +40 & 41 & 18 & Sab35 & $358.6-02.4$ & 17 & 51 & 59.5 & -31 & 18 & 42 \\
\hline Salvi ir & $139.3+04.8$ & 03 & 23 & 01.3 & +62 & 46 & 41 & Sab36ir & $358.7-03.0$ & 17 & 54 & 41.1 & -31 & 31 & $45^{a}$ \\
\hline
\end{tabular}

